\title{
Variable Stiffness Structures Utilizing Pneumatic Artificial Muscles
}

\author{
Ning Feng ${ }^{1}$, Yingli Chang ${ }^{1}$ and Jingze Wang ${ }^{2}$ \\ ${ }^{1}$ Mechanical Engineering Department, Pingdingshan University, 467000 Pingdingshan, China \\ ${ }^{2}$ Institute for Composite Science Innovation, Zhejiang University, 310027 Hangzhou, China
}

\begin{abstract}
Pneumatic artificial muscles (PAMs) can offer excellent force-to-weight ratios and act as shapechanging actuator under injecting the actuation fluid into their bladders. PAMs could be easily utilized for morphing structures due to their millimeter-scale diameter. The pressurized PAM can serve not only as artificial muscle actuator which obtains contraction deformation capability but also as a spring system with variable stiffness. In this study, the stiffness behaviors of pressurized PAMs and a variable stiffness structure are investigated. By taking advantage of the designed PAMs which was conducted by the nonlinear quasi-static model, significant changes in the spring stiffness can be achieved by air pressure control. A case study is presented to explore the potential behavior of a structure with circular permutation PAMs. The structure used in this case consists of sixteen PAMs with circular homogeneous distribution and a circular supporter with sixteen slide way runners. The stiffness of presented structure can vary flexibly in wide range through controlling the air pressure levels and slide deformation respectively.
\end{abstract}

\section{Introduction}

Recently pneumatic artificial muscle (PAM) has received much attention in many application fields of bionics [1,2], medical care, morphing structures [3,4] and welfare, etc. due to its significant advantages of low cost, quick response time and high power/weight and power/volume ratios. Wereley et al. [5,6] employed PAM and millimeter-scale PAM for actuators of morphing structure and small unmanned aerial vehicle (UAV). In 2011 Chen et al. [7] created a morphing skin by embedding pneumatic muscle fibers (PAMs) in a flexible matrix of silicone rubber. In Chen et al.' s study the transverse stiffness of the morphing skin could be regulated by changing the internal air pressure. Larry et al. [8] also fabricated a shape-changing panel with embedded McKibben-like PAMs into neat elastomer. Previously, Feng et al. [9] embedded pneumatic muscle fibers into elastomer to form one kind of single-layer morphing skins. PAMs have shown the feasibility of serving for morphing structures and shape-adaptive structures.

Shan et al. [10] presented a multi-cellular fluidic flexible matrix composites (F2MC) sheet embedding multiple F2MC tubes side by side in a soft matrix. The stiffness ratio of the multi-cellular F2MC sheet was obtained using the isostrain assumption (also known as the Rule of Mixtures) which showed the good agreement with the experimental results. Moreover a case study has been conducted to investigate the behavior of laminated [+60/0/-60]s multi-cellular F2MC sheets [10].
Marouene et al. [11-12] investigated the buckling behavior and the effects of gaps and overlaps on the buckling behavior of variable-stiffness composite laminates. White et al. [13] presented a numerical investigation into the buckling and post-buckling of two variable-stiffness cylinders under axial compression. Kuder et al. [14] explored the design space of two classes of bi-stable composites specially designed to provide stiffness variability in distributed compliance systems. Also many researchers analyzed vibration behaviors of variable stiffness composite laminated plates [15-17].

In this paper, a variable stiffness structure utilizing pneumatic artificial muscles are proposed and investigated. By taking advantage of the designed PAMs and the pressurizing air, significant changes in the spring stiffness can be achieved by air pressure control. A case study is presented to explore the potential behavior of a structure with circular permutation PAMs. The stiffness of presented structure can vary flexibly in wide range through controlling the air pressure levels and slide deformation respectively.

\section{Fabrication and test of PAMs}

We manufactured PAM prototype in house just like the same fabrication process used in Feng et al. [9]. The PAM geometry and bladder material information are listed in Table 1. 
Table 1 Geometrical parameters of PAM.

\begin{tabular}{cccc}
\hline & $\begin{array}{c}\text { Parameter } \\
\text { symbol }\end{array}$ & Description & Value \\
\hline PAM & $L_{i}$ & Initial length & $62.5 \mathrm{~mm}$ \\
\hline \multirow{2}{*}{ Bladder } & $B_{i}$ & Initial outer radius & $2 \mathrm{~mm}$ \\
\cline { 2 - 4 } $\begin{array}{c}\text { Braided } \\
\text { sleeve }\end{array}$ & $A_{i}$ & Initial inner radius & $1 \mathrm{~mm}$ \\
\hline
\end{tabular}

As seen in Fig. 1, the PMF underwent quasi-static testing using a $2000 \mathrm{~N}$ Instron servo-hydraulic test machine (Instron Company, USA) to characterize the performance of PMF. This machine could provide the force measurements; meanwhile the displacement could be regulated smoothly during the testing. An air compressor could provide air supply for PMFs. And a pressure transducer and a pressure governor were used to monitor the air pressure supplied to the PMFs accurately.

Fig. 2 and Fig. 3 show that force-displacement lines of PAM have obvious hysteresis, with the lower line corresponding to the path from output force to free contraction and the upper line corresponding to the return path. The block force increases with the actuation pressure. These experimental data were used to validate the predictions based on the stiffness model of pressurized PMF.

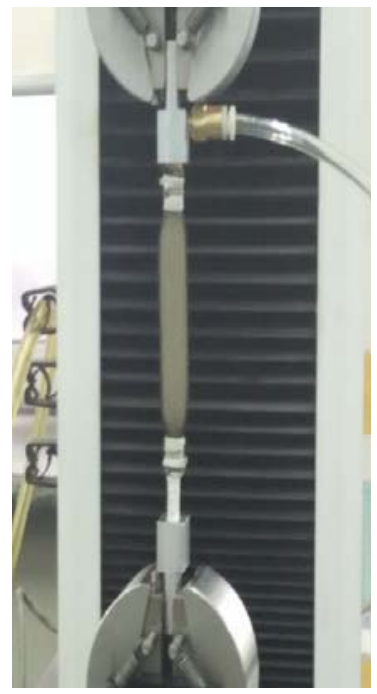

Fig. 1. The experimental setup related to the test of PAM.

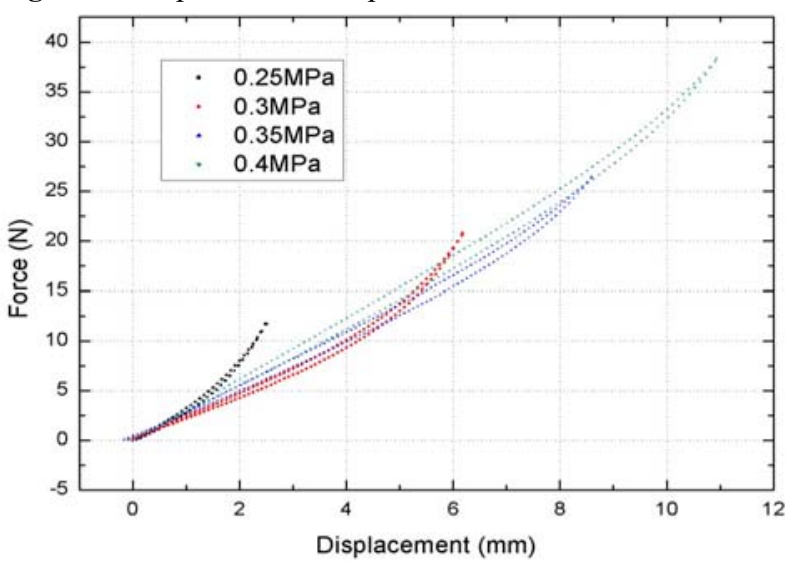

Fig. 2. The experimental results of pressurized PAM.

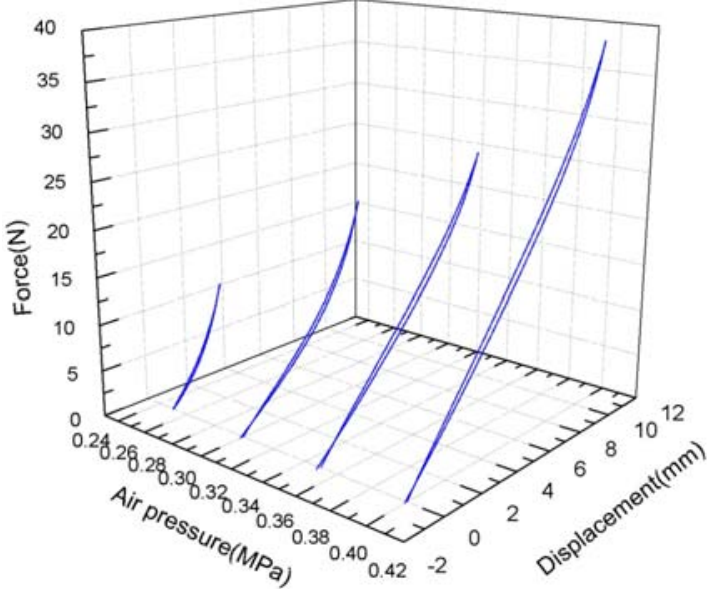

Fig. 3. The experimental results of pressurized PAM in 3-D reference system.

\section{The structure with multiple PAMs}

In this section, a case study is presented to explore the potential behavior of a structure with circular permutation PAMs. The structure used in this case consists of sixteen PAMs with circular homogeneous distribution and a circular supporter with sixteen slide way runners. Fig. 3 shows an illustration of the top view of the structure. Fig. 4 (a) and (b) show that sixteen pressurized PAMs are under limited sliding condition and unlimited sliding condition respectively. PAMs in the following analysis are always actuated and pressurized PAMs can achieve slide deformation in the elastic deformation phase by controlling slide way runners.

Here in order to obtain more accurate stiffness function, the tested force vs. displacement curves of pressurized PAM are fitted by exponential function. Polynomial, trigonometric and exponential function were tried to fit the tested curve respectively, it is found that exponential function is the most convenient choice.

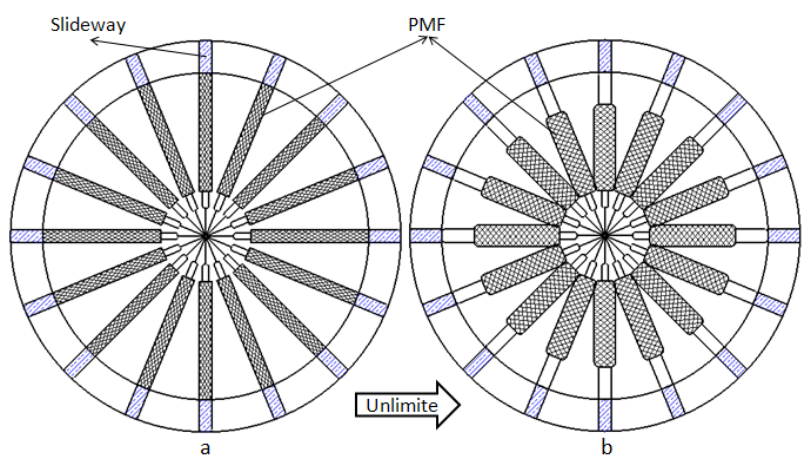

Fig. 4. The structure with multiple PAMs: (a) sixteen pressurized PAMs are under limited sliding condition, (b) sixteen pressurized PAMs are under unlimited sliding condition.

The exponential function used for fitting experimental data is 


$$
F=A_{1} e^{\frac{-z}{t_{1}}}+A_{2} e^{\frac{-z}{t_{2}}}+F_{0}
$$

where the definition of the Eq. 1 is consistent with the Eq. 2. $A_{1}, A_{2}, t_{1}, t_{2}$ and $F_{0}$ are undetermined constants.

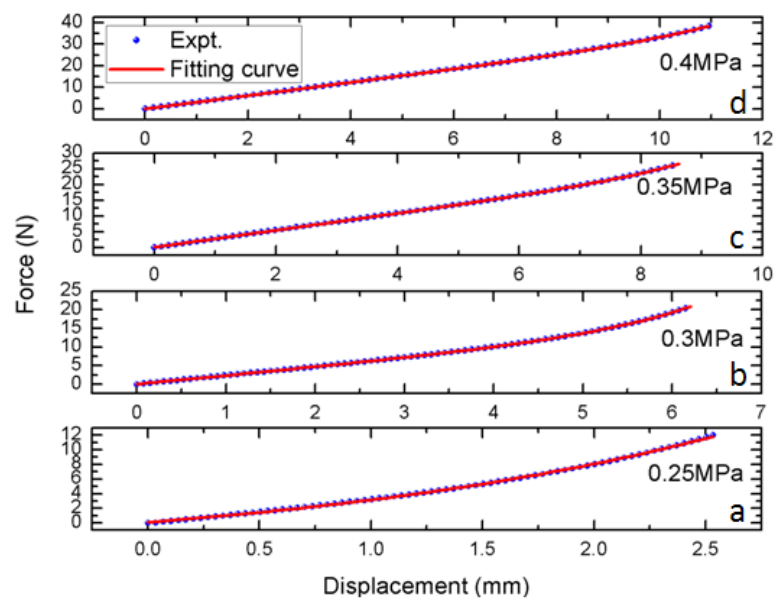

Fig. 5. Comparison of fitted and tested force vs. displacement line responses under different pressure levels: (a) $0.25 \mathrm{MPa}$, (b) $0.3 \mathrm{MPa}$, (c) $0.35 \mathrm{MPa}$, (d) $0.4 \mathrm{MPa}$.

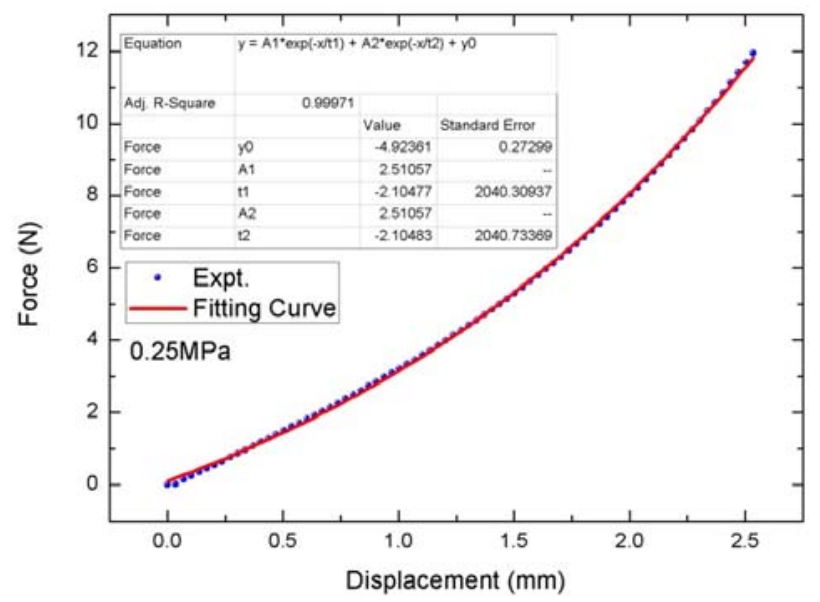

Fig. 6. Comparison of fitted and tested force vs. displacement line responses under $0.25 \mathrm{MPa}$.

As seen in Fig. 5 and 6, the exponential function correlate well with experimental data. The fitting Adj. RSquare are 0.99971, 0.99999, 0.99996 and 1 under different pressure levels, respectively.

We differentiate the Eq. 1, and the stiffness function of pressurized PAM can be obtained as follows

$$
\frac{d F}{d z}=\frac{d f(z)}{d z}=k_{P M F}(z)=-\frac{A_{1}}{t_{1}} e^{\frac{-z}{t_{1}}}-\frac{A_{2}}{t_{2}} e^{\frac{-z}{t_{2}}}
$$

Through the calculation of the Eq. 2, apparent stiffness constant of single pressurized PAM and the structure with multiple pressurized PAMs can be obtained.

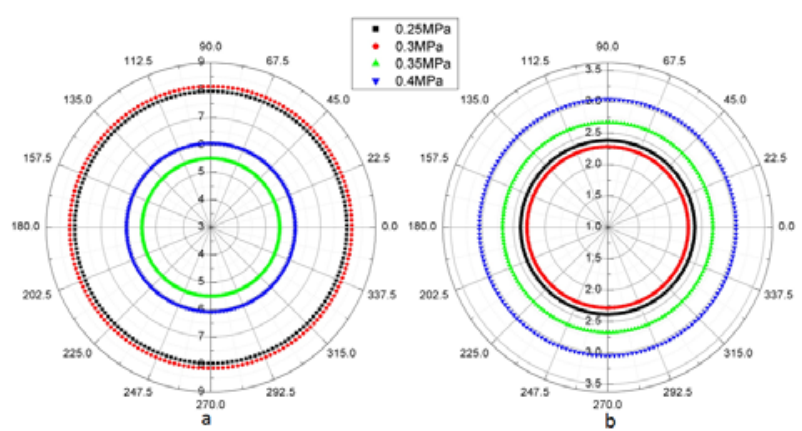

Fig. 7. Polar plots of the apparent stiffness of the structure with multiple PAMs as function of orientation. The radial direction represents the stiffness constant, where the unit of stiffness constant is N/mm: (a) sixteen pressurized PAMs are under limited sliding condition, (b) sixteen pressurized PAMs are under free sliding condition.

Through the calculation of the equation 2, apparent stiffness constant of single pressurized PAM and the structure with multiple pressurized PAMs can be obtained. Figure 7 shows polar plots of the apparent stiffness of the structure with multiple PAMs as function of orientation. The radial direction represents the stiffness constant, where the unit of stiffness constant is $\mathrm{N} / \mathrm{mm}$. As seen in Fig. 7(a), the maximum and minimum stiffness constant are $8.13 \mathrm{~N} / \mathrm{mm}$ and $5.51 \mathrm{~N} / \mathrm{mm}$ respectively when sixteen pressurized PAMs are under limited sliding condition. As seen in Fig. 7(b), the maximum and minimum stiffness constant are $3.04 \mathrm{~N} / \mathrm{mm}$ and $2.28 \mathrm{~N} / \mathrm{mm}$ respectively when sixteen pressurized PAMs are under free sliding condition. It means the stiffness change of this present structure can reach $148 \%$ or $133 \%$ only through controlling the air pressure levels when sixteen pressurized PAMs are under limited sliding condition or free sliding condition. Moreover the stiffness change of this present structure can reach up to $357 \%$ though controlling the air pressure levels and slide deformation of pressurized PAMs in the slide way runners.

In the previous discussion, the variable stiffness capability of the structure is achieved by controlling sixteen pressurized PAMs which are under same air pressure level and under same slide deformation in the slide way runners. Fig. 8 shows that the present structure is capable of varying stiffness partially if we control sixteen pressurized PAMs which are under different air pressure levels and different slide deformation. Here the pressurized PAMs in first and third quartiles are controlled under $0.3 \mathrm{MPa}$ and limited sliding condition; the pressurized PAMs in second and fourth quartiles are controlled under $0.3 \mathrm{MPa}$ and free sliding condition. In this way the stiffness behaviors of this structure are similar to the orthotropic laminate. Obviously the stiffness of this presented structure can vary more flexibly in wider range if sixteen pressurized PAMs are controlled under different air pressure levels and different slide deformation respectively. 


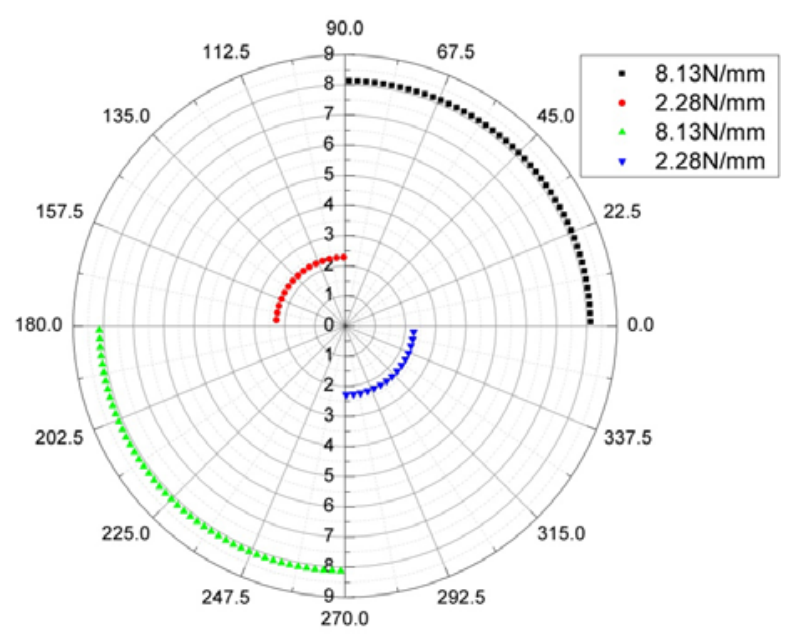

Fig. 8. Polar plots of the partial variable stiffness of the structure with multiple PAMs as function of orientation.

\section{Summary}

In this study, the pressurized PAM is investigated as a spring system with variable stiffness. The non-linear quasi-static model successfully captured block force and displacement of pressurized PMF. This model can be used to conduct the PMF design, especially for the spring stiffness design. A case study is presented to explore the potential behaviors of a structure with circular permutation PAMs. When sixteen pressurized PAMs are under limited sliding condition or free sliding condition, the stiffness change of this structure can reach $148 \%$ or $133 \%$ only through controlling the air pressure levels. The stiffness change of this structure can reach up to $357 \%$ through controlling the air pressure levels and slide deformation of pressurized PAMs. Moreover, the abovementioned structure is capable of varying stiffness partially when sixteen pressurized PAMs are under different air pressure levels and different slide deformation.

\section{References}

1. P. Ohta, L.Valle, J. King, et al, Design of a Lightweight Soft Robotic Arm Using Pneumatic Artificial Muscles and Inflatable Sleeves. Soft Robotics, (2017)

2. Y. Cui, T. Matsubara, K. Sugimoto, Pneumatic artificial muscle-driven robot control using local update reinforcement learning. Advanced Robotics, 31(8): 397-412, (2017)

3. T. E. Pillsbury, N. M. Wereley, Q. Guan, Comparison of contractile and extensile pneumatic artificial muscles. Smart Materials and Structures, 26(9): 095034, (2017).

4. B. K. S. Woods, O. Bilgen, M. I. Friswell, Wind tunnel testing of the fish bone active camber morphing concept. Journal of Intelligent Material Systems and Structures, 25(7): 772-785, (2014).
5. B. K. S. Woods, M. F. Gentry, C. S. Kothera, N. M. Wereley, Fatigue life testing of swaged pneumatic artificial muscles as actuators for aerospace applications. Journal of Intelligent Material Systems and Structures, 23(3): 327-343, (2012)

6. E. G. Hocking, N. M. Wereley, Analysis of nonlinear elastic behavior in miniature pneumatic artificial muscles. Smart Materials and Structures, 22(1): 014016, (2013).

7. Y. Chen, W. Yin, Y. Liu, J. Leng, Structural design and analysis of morphing skin embedded with pneumatic muscle fibers. Smart Materials and Structures, 20(8): 085033, (2011)

8. L. D. Peel, J. W. Baur, R. S. Justice, Characterization and application of shape-changing panels with embedded rubber muscle actuators. Smart Materials and Structures, 22(9): 094020, (2013).

9. N. Feng, L. Liu, Y. Liu, J. Leng. A bio-inspired, active morphing skin for camber morphing structures. Smart Materials and Structures, 24(03): 035023, (2015)

10. Y. Shan, M. Philen, A. Lotfi, Variable stiffness structures utilizing fluidic flexible matrix composites. Journal of Intelligent Material Systems and Structures, 20(4): 443-456, (2009)

11. A. Marouene, R. Boukhili, J. Chen, Buckling behavior of variable-stiffness composite laminates manufactured by the tow-drop method. Composite Structures, 139: 243-253, (2016)

12. A. Marouene, R. Boukhili, J. Chen, Effects of gaps and overlaps on the buckling behavior of an optimally designed variable-stiffness composite laminates - A numerical and experimental study. Composite Structures, 140: 556 - 566, (2016)

13. S. C. White, P. M. Weaver, K. C. Wu, Post-buckling analyses of variable-stiffness composite cylinders in axial compression. Composite Structures, 123: 190203, (2015)

14. I. K. Kuder, A. F. Arrieta, P. Ermanni, Design space of embeddable variable stiffness bi-stable elements for morphing applications. Composite Structures, 122: 445-455, (2015)

15. P. Ribeiro, H. Akhavan, Non-linear vibrations of variable stiffness composite laminated plates. Composite Structures, 94(8): 2424-2432, (2012)

16. H. Akhavan, P. Ribeiro, Natural modes of vibration of variable stiffness composite laminates with curvilinear fibers. Composite Structures, 93(11): 3040-3047, (2011)

17. M. M. Abdalla, S. Setoodeh, Z. Gürdal, Design of variable stiffness composite panels for maximum fundamental frequency using lamination parameters. Composite structures, 81(2): 283-291, (2007) 